\title{
Spherical Extension Property No Longer True for Domains in Algebraic Variety with Isolated Singularity
}

\author{
Shanyu Ji, Stephen S.T. Yau, and Cheng Zhan
}

September 25, 2009

Dedicated to Professor Lo Yang on the occasion of his 70th birthday

\section{Introduction}

Let $D$ be a domain in $\mathbb{C}^{n}$. By $\partial D$ spherical, we mean that for each point $p \in \partial D$, there is a biholomorphic map $f: U \rightarrow V$ such that $f(U \cap \partial D) \subseteq V \cap \partial \mathbb{B}^{n}$ where $U, V$ are some open subsets of $\mathbb{C}^{n}$ with $p \in U$ and $f(p) \in V$. It is known ([CJ96]) that if $D$ is a simply connected bounded domain in $\mathbb{C}^{n}$ with spherical real analytic boundary $\partial D$, then every local biholomorphic map at boundary as above extends to a biholomorphic map from $D$ onto $\mathbb{B}^{n}$. As a consequence, a local biholomorphic map between $\partial D_{1}$ and $\partial D_{2}$ where $D_{1}, D_{2}$ are simply connected domains in $\mathbb{C}^{n}$ with spherical real analytic boundaries can extend to a global biholomorphic map from $D_{1}$ onto $D_{2}$. If the boundary is algebraic, the simply connected condition in the above result can be dropped (cf. [HJ98]).

In this short note, we want to show that the above phenomenon is no longer true if domains are in algebraic varieties with isolated singularities.

Theorem 1.1 There are two distinct simply connected domains $E_{j} \subset A$ with spherical algebraic boundaries $\partial E_{j}, j=1,2$, where $A$ is a 2 complex dimensional algebraic variety in $\mathbb{C}^{3}$ with one isolated singularity, and there is a local biholomorphic map $\widetilde{F}: \widetilde{U}_{1} \rightarrow \widetilde{U}_{2}$ with $\widetilde{F}\left(\widetilde{U}_{1} \cap \partial E_{1}\right) \subset \widetilde{U}_{2} \cap \partial E_{2}$ where $\widetilde{U}_{j}$ are open subsets in $A$ with $\widetilde{U}_{j} \cap \partial E_{j} \neq \emptyset, j=1,2$, such that $\widetilde{F}$ cannot extend to a biholomorphic map from $E_{1}$ onto $E_{2}$.

The algebraic variety $A$ in Theorem 1.1 is constructed as follows. Let $\Gamma$ be a cyclic group of order two acting on $\mathbb{C}^{2}$, sending $(x, y)$ to $(-x,-y)$. Let

$$
A=\left\{\left(w_{1}, w_{2}, w_{3}\right) \in \mathbb{C}^{3} \mid w_{1} w_{2}=w_{3}^{2}\right\}
$$


be algebraic variety in $\mathbb{C}^{3}$ with one isolated singularity $(0,0,0)$. Let

$$
\begin{array}{cccc}
L: & \mathbb{C}^{2} & \rightarrow & A \subset \mathbb{C}^{3} \\
& \left(z_{1}, z_{2}\right) & \mapsto & \left(L_{1}, L_{2}, L_{3}\right)=\left(z_{1}^{2}, z_{2}^{2}, z_{1} z_{2}\right)
\end{array}
$$

be a holomorphic map. The functions $L_{1}, L_{2}$ and $L_{3}$ are invariant under the action of $\Gamma$. We define an equivalent relation for points in $\mathbb{C}^{2}:\left(z_{1}, z_{2}\right) \sim\left(\widetilde{z}_{1}, \widetilde{z}_{2}\right)$ if and only if either $\left(z_{1}, z_{2}\right)=\left(\widetilde{z}_{1}, \widetilde{z}_{2}\right)$ or $\left(z_{1}, z_{2}\right)=\left(-\widetilde{z}_{1},-\widetilde{z}_{2}\right)$. We see $L\left(z_{1}, z_{2}\right)=L\left(\widetilde{z}_{1}, \widetilde{z}_{2}\right)$ if and only if $\left(z_{1}, z_{2}\right) \sim\left(\widetilde{z}_{1}, \widetilde{z}_{2}\right)$. Thus $L: \mathbb{C}^{2} \rightarrow A$ is the quotient map identifying the quotient space $\mathbb{C}^{2} / \sim$ with $A$.

We notice that by the algebraicity theorem of S. M. Webster [W77] and X. Huang [H94] the map $\widetilde{F}$ in Theorem 1.1 always has a multiple-valued extension without branching points in the boundary.

Corollary 1.2 Let $F: D_{1} \rightarrow D_{2}$ be a biholomorphic map where $D_{j}$ are domains in $\mathbb{C}^{2}$ with $(0,0) \in D_{j}, j=1,2$. Let $E_{j}=L_{j}\left(D_{j}\right)$ and $L_{j}, j=1,2$, be as in (1). If a locally defined map $\widetilde{F}=L_{2} \circ F \circ L_{1}^{-1}$ over an open subset $\widetilde{U}$ of $E_{1}$ can extend holomorphically to a biholomorphic map $\widetilde{F}: E_{1} \rightarrow E_{2}$. Then $F(-z)=-F(z)$ holds for any $z \in \mathbb{B}^{2}(0, r) \subset D_{1}$ where $\mathbb{B}^{2}(0, r)$ is some ball in $\mathbb{C}^{2}$ centered at $(0,0)$ with radius $r$.

Corollary 1.3 Let $D_{2} \subset \mathbb{C}^{2}$ be a strongly pseudoconvex bounded domain with algebraic boundary, such that $(0,0) \in D_{2}$ and it admits the group action of $\Gamma$. Let $F_{0}\left(z_{1}, z_{2}\right)=$ $\left(z_{1}, z_{2}+h\left(z_{1}\right)\right)$ where $h$ is a holomorphic polynomial of $z_{1}$ such that $h\left(-z_{1}\right)=-h\left(z_{1}\right), \forall z_{1}$. Let $D_{1}=F_{0}^{-1}\left(D_{2}\right)$. Suppose that there exists an automorphism $\Phi \in \operatorname{Aut}\left(D_{2}\right)$ with $\Phi(0) \neq 0$. Let $F=\Phi \circ F_{0}$. Then

(i) $\partial E_{1}$ and $\partial E_{2}$ are strongly pseudoconvex bounded domains with algebraic boundaries;

(ii) any locally defined map $\widetilde{F}=L_{2} \circ F \circ L_{1}^{-1}: \widetilde{U}_{1} \rightarrow \widetilde{U}_{2}$ with $\widetilde{F}\left(\widetilde{U}_{1} \cap \partial E_{1}\right) \subset \widetilde{U}_{2} \cap \partial E_{2}$ where $\widetilde{U}_{j}$ are open subsets in $A$ with $\widetilde{U}_{j} \cap \partial E_{j} \neq \emptyset, j=1,2$, cannot extend to a biholomorphic map from $E_{1}$ onto $E_{2}$. 


\section{A domain $D$ and its associated domain $E$}

Let $D$ be a bounded domain in $\mathbb{C}^{2}$ containing the origin, and $E:=L(D)$ be the associated domain in $A$.

Let $\partial D=\{z \mid \rho(z, \bar{z})=0\}$ be a real analytic hypersurface in $\mathbb{C}^{2}$ where $\rho$ is the defining function. In general, $\partial E$ may not be smooth. In fact, let $z \in \partial D$ and let us consider the following cases.

(ia) If $-z \notin \partial D$ with $-z \in D$, then $L(z)$ is not a boundary point of $E$.

(ib) If $-z \notin \partial D$ with $-z \notin D$, then the boundary $\partial E$ is smooth and real analytic at $L(z)$.

(iia) If $-z \in \partial D$ with

$$
L\left(U_{z} \cap \partial D\right)=L\left(U_{-z} \cap(-\partial D)\right)
$$

where $U_{z}$ and $U_{-z}$ are some neighborhoods of $z$ and $-z$ in $\mathbb{C}^{2}$ respectively, $\partial E$ is real analytic at $L(z)$.

(iib) If $-z \in \partial D$ such that $L\left(U_{z} \cap \partial D\right)=L\left(U_{-z} \cap(-\partial D)\right)$ does not hold for any $U_{z}$ and $U_{-z}$, then $\partial E$ may not be smooth at $L(p)$.

Let $D \subset \mathbb{C}^{2}$ be a bounded domain with connected real analytic boundary $\partial D$ and $0 \in D$. By the uniqueness of real analytic functions, (iia) holds at a point in $\partial D$ if and only if (iia) holds for all points at $\partial D$. Notice that $\Lambda:=\partial D \cap(-\partial D) \neq \emptyset$ always holds because $D$ contains $(0,0)$. Then (ia) or (ib) cannot hold for all points of $\partial D$. So, either (iia) holds for all points in $\partial D$; or (ia) (or (ib)) holds for majority of points of $\partial D$ and (iib) holds on a proper real analytic subvariety of $\partial D$. In the case (iia) for all points, the boundary $\partial E$ is real analytic. In the case (ia)(iib) or (ib)(iib) hold, the boundary $\partial E$ may not be smooth. For example, the property (iia) holds for the domain $D=\mathbb{B}^{2}=\left\{\left.\left(z_{1}, z_{2}\right)|| z_{1}\right|^{2}+\left|z_{2}\right|^{2}<1\right\}$, and (iib) holds for the domain $D=\left\{\left(z_{1}, z_{2}\right)|| z_{1}-\left.1\right|^{2}+\left|z_{2}\right|^{2}<4\right\}$ in which $\partial E$ is not smooth at the point $L(0, \sqrt{3})=L(0,-\sqrt{3})=(0,3,0)$.

\section{Proof of Theorem}

Proof of Theorem: Let $D_{1}=D_{2}=\mathbb{B}^{2}(0,1)$ be the unit ball in $\mathbb{C}^{2}$. Let $E_{1}=L\left(D_{1}\right)$ and $E_{2}=L\left(D_{2}\right)$ be the induced domains in $A . E_{j}=L\left(\mathbb{B}^{2}(0,1)\right)$ is simply connected because it is contractable by the $C^{*}$ action. By the way, its boundary $\partial E_{j}$ is not simply connected in view of Mumford's theorem [M61] because the interior singularity is normal. Since $D_{1}$ and $D_{2}$ are invariant under the $\Gamma$ action, $\partial E_{1}$ and $\partial E_{2}$ are smooth, algebraic and spherical. 
We take an automorphism $F \in A u t\left(\mathbb{B}^{n}\right)$ satisfying

$$
F(0) \neq 0 .
$$

Now $F$ induces a local biholomorphically equivalence map $\widetilde{F}:=L_{2} \circ F \circ L_{1}^{-1} . \widetilde{F}: \widetilde{U}_{1} \rightarrow \widetilde{U}_{2}$ which is a biholomorphic map such that $\widetilde{F}\left(\partial E_{1} \cap \widetilde{U}_{1}\right) \subseteq \partial \widetilde{E}_{2} \cap U_{2}$, where $\widetilde{U}_{1}$ and $\widetilde{U}_{2}$ are open subsets in $A-\{(0,0,0)\}$. Conversely, the map $F$ can be recovered by $\left.F\right|_{U_{1}}=L_{2}^{-1} \circ \widetilde{F} \circ L_{1}$ that is a biholomorphic map from $U_{1} \subset D_{1}-\{(0,0)\}$ onto $U_{2} \subset D_{2}-\{(0,0)\}$ where $L_{1}\left(U_{1}\right)=\widetilde{U}_{1}, L_{2}\left(U_{2}\right)=\widetilde{U}_{2}$.

Suppose that the map $\widetilde{F}$ can extend to a biholomorphic map $\widetilde{F}: E_{1} \rightarrow E_{2}$. We want to find a contradiction.

Consider the following points

$$
z_{0}=\left(z_{01}, z_{02}\right) \in U_{1}, \widetilde{z}_{0}:=L_{1}\left(z_{0}\right)=\left(\left(z_{01}\right)^{2},\left(z_{02}\right)^{2}, z_{01} z_{02}\right) \in \widetilde{U}_{1}, \widetilde{w}_{0}:=\widetilde{F}\left(\widetilde{z}_{0}\right) \in \widetilde{U}_{2} .
$$

Take a closed curve in $E_{1}$ :

$$
\widetilde{\theta}(t)=\left(\left(z_{01}\right)^{2} e^{2 i \pi t},\left(z_{02}\right)^{2} e^{2 i \pi t}, z_{01} z_{02} e^{4 i \pi t}\right) \in E_{1}-\{(0,0,0)\}, t \in[0,1] .
$$

Here $\widetilde{\theta}(0)=\widetilde{z}_{0}$. There is a unique lifting curve $\theta$ in $D_{1}$ of the curve $\widetilde{\theta}$ such that $\theta(0)=z_{0}$, namely,

$$
\theta(t)=L_{1}^{-1}(\widetilde{\theta}(t))=\left(z_{01} e^{i \pi t}, z_{02} e^{i \pi t}\right), \quad \forall t \in[0,1] .
$$

Since $z_{0} \neq 0$, the curve $\theta$ is not closed:

$$
\theta(0)=z_{0} \quad \text { and } \quad \theta(1)=-z_{0} .
$$

Since $\widetilde{\theta}$ is a closed curve and $\widetilde{F}$ is biholomorphic, we have $\widetilde{F}(\widetilde{\theta}(0))=\widetilde{F}(\widetilde{\theta}(1))$ so that the curve $\widetilde{\psi}=\widetilde{F} \circ \widetilde{\theta}$, with $\widetilde{\psi}(0)=\widetilde{F}\left(\widetilde{z}_{0}\right)=\widetilde{w}_{0}$, is also a closed curve in $E_{2}$. We notice that if $\widetilde{F}$ is not a globally defined map, we cannot conclude that $\widetilde{\psi}$ is closed.

Because $\widetilde{F}$ is biholomorphic map, $\widetilde{F}$ sends smooth points of $A$ to smooth points of $A$. Therefore, the curve $\widetilde{\psi}$ does not intersect $(0,0,0)$.

Away from the point $(0,0,0), L_{2}^{-1}$ is locally defined. By the uniqueness property of holomorphic functions, the map $F=L_{2}^{-1} \circ \widetilde{F} \circ L_{1}$ must hold (by holomorphic extension of $\left.\left.F\right|_{U_{1}}\right)$ along the curve $\theta$, namely, $\left.F\right|_{\theta(t)}=\left.L_{2}^{-1} \circ \widetilde{F} \circ L_{1}\right|_{\theta(t)}$. Then we have

$$
F\left(-z_{0}\right)=F(\theta(1))=L_{2}^{-1} \circ \widetilde{F} \circ L_{1}(\theta(1)) \in L_{2}^{-1} \circ \widetilde{\psi}(1),
$$

and

$$
F\left(z_{0}\right)=F(\theta(0))=L_{2}^{-1} \circ \widetilde{F} \circ L_{1}(\theta(0)) \in L_{2}^{-1} \circ \widetilde{\psi}(0) .
$$


Since $\widetilde{\psi}$ is closed, $\widetilde{\psi}(0)=\widetilde{\psi}(1)$ so that $L_{2}^{-1} \circ \widetilde{\psi}(1)=L_{2}^{-1} \circ \widetilde{\psi}(0)=L_{2}^{-1}\left(\widetilde{w}_{0}\right)$.

Since $F\left(z_{0}\right) \in L_{2}^{-1}\left(\widetilde{w}_{0}\right)$, by the definition of $L_{2}$, we see $L_{2}^{-1}\left(\widetilde{w}_{0}\right)=\left\{F\left(z_{0}\right),-F\left(z_{0}\right)\right\}$ so that there are two possibilities:

(i) $F\left(-z_{0}\right)=F\left(z_{0}\right)$;

(ii) $F\left(-z_{0}\right)=-F\left(z_{0}\right)$.

Since $F$ is one-to-one, (i) cannot occur. Then we must have $F\left(-z_{0}\right)=-F\left(z_{0}\right)$.

If we replace $z_{0}$ by any point $z$ in a sufficiently small neighborhood of $z_{0}$, we can use the above argument to imply that $F(-z)=-F(z)$. By the uniqueness,

$$
F(-z)=-F(z), \quad \forall z \in D_{1} .
$$

In particular, it implies $F(0)=-F(0)$ and hence $F(0)=0$, but this is a contradiction with $(3)$.

By similar proof, we can show Corollaries. Notice that there is no boundary condition needed in Corollary 1.2.

\section{References}

[CJ96] S.-S. Chern and S. Ji, On the Riemann mapping theorem, Ann. of Math., 144(1996), 421-439.

[H94] Huang, Xiao Jun On the mapping problem for algebraic real hypersurfaces in the complex spaces of different dimensions. Ann. Inst. Fourier (Grenoble) 44 (1994), no. 2, $433-463$.

[HJ98] X. Huang and S. Ji, Global holomorphic extension of a local map and a Riemann mapping theorem for algebraic domains, Math. Research Letter, 5(1998), 247-260.

[M61] Mumford, David, The topology of normal singularities of an algebraic surface and a criterion for simplicity. Inst. Hautes tudes Sci. Publ. Math. No. 91961 5-22.

[W77] Webster, S. M., On the mapping problem for algebraic real hypersurfaces, Invent. Math. 43 (1977), 53-68.

Shanyu Ji, Department of Mathematics, University of Houston, TX 77204, USA (shanyuji @math.uh.edu) 
Stephen S.-T. Yau, Institute of Mathematics, East China of Normal University, Shanghai, China; current address: MSCS, M/C 249, SED Rm 322, University of Illinois at Chicago, 851, IL 60607-7045, USA (yau@uic.edu)

Cheng Zhan, Department of Mathematics, University of Houston, TX 77204, USA (zhan0511@math.uh.edu) 